\title{
A New Asymptotic Treatment of $g$-modes of a Star
}

\author{
P. Smeyers, T. Van Hoolst, I. De Boeck ${ }^{1}$ and L. Decock \\ Instituut voor Sterrenkunde, Katholieke Universiteit Leuven, \\ Celestijnenlaan 200B, 3001 Heverlee, Belgium
}

\section{Introduction and basic equations}

An asymptotic representation of low-frequency, linear, isentropic $g$-modes of a star is developed without the usual neglect of the Eulerian perturbation of the gravitational potential. Our asymptotic representation is based on the use of asymptotic expansions adequate for solutions of singular perturbation problems (see, e.g., Kevorkian \& Cole 1981).

Linear, isentropic oscillation modes with frequency different from zero are governed by a fourth-order system of linear, homogeneous differential equations in the radial parts of the radial displacement $\xi(r)$ and the divergence $\alpha(r)$. These equations take the form

$$
\begin{aligned}
& \frac{d^{2} \alpha}{d r^{2}}+K_{2}(r) \frac{d \alpha}{d r}+\left[\frac{\sigma^{2}}{c^{2}}+K_{3}(r)+\frac{K_{1}(r)}{\sigma^{2}}\right] \alpha=-K_{4}(r) \frac{d \xi}{d r}, \\
& \frac{d^{2} \xi}{d r^{2}}+\frac{4}{r} \frac{d \xi}{d r}-\frac{\ell(\ell+1)-2}{r^{2}} \xi=\frac{d \alpha}{d r}-\left[\frac{c^{2}}{g} \frac{K_{1}(r)}{\sigma^{2}}-\frac{2}{r}\right] \alpha .
\end{aligned}
$$

The symbols have their usual meaning. $N^{2}$ is the square of the frequency of Brunt-Väisälä. The functions $K_{1}(r), K_{2}(r), K_{3}(r), K_{4}(r)$ depend on the equilibrium model, e.g.,

$$
K_{1}(r)=\ell(\ell+1) \frac{N^{2}}{r^{2}}
$$

We introduce the small expansion parameter

$$
\epsilon=|\sigma| \text {. }
$$

and assume, for the sake of simplification, $N^{2}$ to be positive everywhere in the star so that the star is everywhere convectively stable.

\section{Method}

Because of the singularities appearing in the differential equations at the centre and the surface of the star, it is necessary to make a distinction between the region sufficiently far from both singular points, and the two regions near the singular points.

\footnotetext{
${ }^{1}$ Research Assistant of the Belgian National Fund for Scientific Research
} 
At distances sufficiently large from the star's centre and surface, we consider Eq. (1) as a differential equation governing a linear oscillator with a high frequency and a small damping. By using a two-variable expansion procedure, we seek asymptotic solutions in terms of a fast and a slow variable in the radial direction. Next, we seek solutions for the other second-order differential equation in terms of the same variables.

We treat the regions near the centre and the surface of the star as boundary layers.

By matching the appropriate asymptotic expansions and imposing the continuity of the gravitational potential and its gradient, we derive the equation determining the eigenfrequencies, and the various constants involved in the solutions.

\section{Conclusions}

- The Cowling approximation $\left(\Phi^{\prime}=0\right)$ is valid in the lowest-order asymptotic approximation. The same eigenfunctions and eigenfrequencies are found as previously derived in the Cowling approximation (see, e.g., Smeyers \& Tassoul 1987).

- The subseismic approximation $\left(P^{\prime}=0\right)$ is valid from the centre to a distance sufficiently far from the surface in the lowest-order asymptotic approximation.

- The relative order of the lowest-order approximations of the eigenfunctions of $g$-modes in the boundary layer near $r=0$ (central), the outer region, and the boundary layer near $r=R$ (surface) are given in the table below. When the lowest-order approximation is equal to zero, the lowest, possibly non-zero order is indicated between braces.

\begin{tabular}{lccc}
\hline & central & outer & surface \\
\hline$\alpha / \xi$ & $\varepsilon$ & $\varepsilon^{0}$ & $\varepsilon^{-2}$ \\
$\eta / \xi$ & $\varepsilon$ & $\varepsilon^{-1}$ & $\varepsilon^{-2}$ \\
$\rho^{\prime} /(\rho \xi)$ & $\varepsilon$ & $\varepsilon^{0}$ & $\varepsilon^{-2}$ \\
$P^{\prime} /(\rho \xi)$ & $\left(\varepsilon^{3}\right)$ & $(\varepsilon)$ & $\varepsilon^{0}$ \\
$\Phi^{\prime} / \xi$ & $\left(\varepsilon^{3}\right)$ & $(\varepsilon)$ & $\left(\varepsilon^{2}\right)$ \\
\hline
\end{tabular}

Full details will be published in Astronomy and Astrophysics.

\section{References}

Kevorkian, J., \& Cole, J.D. 1981, Perturbation Methods in Applied Mathematics, Springer, New-York

Smeyers, P., \& Tassoul, M. 1987, ApJS, 65, 429 